\title{
ON THE NORMABILITY OF THE INTERSECTION OF $L_{p}$ SPACES
}

\author{
WAYNE C. BELL
}

\begin{abstract}
The set $L_{\omega}=\bigcap_{p=1}^{\infty} L_{p}[0,1]$ is not equal to $L_{\infty}[0,1]$ since $L_{\omega}$ contains the function $-\ln x$. Using the theory of $L_{p}$ spaces for finitely additive set functions developed by Leader [9] we will prove several necessary and sufficient conditions for the normability of a generalization of $L_{\omega}$. These include the equality and finite dimensionality of all the $L_{p}$ spaces, $p>1$.
\end{abstract}

1. Introduction. The set $L_{\omega}=\bigcap_{p=1}^{\infty} L_{p}[0,1]$ is not equal to $L_{\infty}[0,1]$ since $L_{\omega}$ contains the function $-\ln x$. In [2] Arens showed that with the natural $\omega$-topology, i.e. the smallest topology which contains each relative $p$-norm topology, $L_{\omega}$ is not normable. In [5] and [6] Davis, Murray and Weber discussed $L_{p}-(\mu)=\bigcap_{q<p} L_{q}(\mu)$ which, when $p=\infty$ and $\mu$ is Lebesgue measure on $[0,1]$, reduces to $L_{\omega}$. Using the theory of $L_{p}$ spaces for finitely additive set functions developed by Leader [9], we will prove several necessary and sufficient conditions for the normability of a generalization of $L_{\omega}$. These include the equality and finite dimensionality of all the $L_{p}$ spaces, $p \geqslant 1$.

Suppose $S$ is a set, $F$ a field of subsets of $S$ and ba $(F)$ the set of bounded finitely additive functions from $F$ into the real numbers. If $G \subseteq \mathrm{ba}(F)$, then $G^{+}$will denote the nonnegative valued elements of $G . A_{\lambda}$ will be the set of elements of $\mathrm{ba}(F)$ which are absolutely continuous with respect to $\lambda \in$ $\mathrm{ba}(F)^{+}$. It should be noted that we mean absolutely continuous in the $\varepsilon-\delta$ sense which is (for $\mathrm{ba}(F)$ ) stronger than the $0-0$ sense. Henceforth, $\mu$ will be in $\mathrm{ba}(F)^{+}$with $\mu(S)=1$. We adopt the convention that $a / 0$ is 0 .

2. " $L_{p}$ spaces" for $\mu$ and $H_{\omega}(\mu)$. For $1 \leqslant p<\infty$ we define $H_{p}(\mu)$ to be the set to which $\xi$ belongs iff

(1) $\xi \in A_{\mu}$ and

(2) there exists an $M>0$ such that $\Sigma_{D}|\xi(I)|^{p} \mu(I)^{1-p}<M$ for each (finite) subdivision $D$ of $S$ by elements of $F$.

If $p>1$, then (1) may be replaced by: $\mu(I)=0$ implies $|\xi(I)|=0$. If $p=2$

Presented to the Society, December 23, 1974 under the title Some normability conditions for $H_{\mu \omega}$; received by the editors February $1,1977$.

AMS (MOS) subject classifications (1970). Primary 46E30; Secondary 28A10, 28A25, 46A05.

$K e y$ words and phrases. Finitely additive set function, $L_{p}$ spaces, normability.

${ }^{1}$ The author wishes to express his appreciation to Professor W. D. L. Appling who directed the dissertation from which this paper was taken. 
we have the Hilbert space of Hellinger integrable functions which, when $F$ is a $\sigma$-field and $\mu$ is countably additive, corresponds to $L_{2}(\mu)$. The $p$-norm $(1 \leqslant p<\infty)$ denoted, $\|\cdot\|_{p}$, is the $p$ th root of the supremum of the sums in (2). $H_{\infty}(\mu)$ will be $\left\{\xi \in A_{\mu}|| \xi(I) \mid \mu(I)^{-1}\right.$ is bounded on $\left.F\right\}$ (the $\operatorname{Lip}(\mu)$ of [4]) with $\|\xi\|_{\infty}=\sup \left\{|\xi(I)| \mu(I)^{-1} \mid I \in F\right\}$. With this norm $H_{p}(\mu)(1 \leqslant p \leqslant \infty)$ is a Banach space [9]. If $p=1$, then $H_{p}(\mu)$ is $A_{\mu}$ with the total variation norm.

For $\xi, \eta$ and $\delta$ in $\operatorname{ba}(F)$ we will denote by $\eta \wedge \delta$ the element of $\operatorname{ba}(F)$ whose value at $v \in F$ is $\sup \{g(v) \mid g \in \mathrm{ba}(F), g \leqslant \eta$ and $g \leqslant \delta\}, \eta \vee \delta$ will be $-[(-\eta) \wedge(-\delta)]$ and $\xi \vee 0$ will be written $\xi^{+}$, so that the total variation function for $\xi$ will be $|\xi|=\xi^{+}+\xi^{-}$, where $\xi^{-}=(-\xi)^{+}$.

In the interest of self-containment we state some of the results from [9] which we will need.

TheOREM 2. L. Suppose each of $\eta$ and $\delta$ is in $A_{\mu}, \lambda \in A_{\mu}^{+}$and $1 \leqslant q \leqslant p \leqslant$ $\infty$. Then we have;

I. If $|\delta| \leqslant|\eta|$, then $\eta \in H_{p}(\mu)$ iff $|\eta| \in H_{p}(\mu)$, in which case $\delta \in H_{p}(\mu)$ and $\|\delta\|_{p} \leqslant\|\eta\|_{p}=\||\eta|\|_{p}$.

II. The sequence $(\lambda \wedge K \mu)_{K=1}^{\infty}$ is $p$-norm convergent to $\lambda$ iff $\lambda \in H_{p}(\mu)$ and, therefore, $H_{\infty}(\mu)$ is $p$-norm dense in $H_{p}(\mu)$.

III. The strength of the p-norms is nondecreasing with $p$ and therefore

$$
H_{\infty}(\mu) \subseteq H_{p}(\mu) \subseteq H_{q}(\mu) \subseteq A_{\mu} .
$$

Suppose $\lambda \in A_{\mu}^{+}, 1<p<\infty, D$ is a subdivision of $S$ and $N$ is the number of elements in $D$. Then there exists a $K \in \mathbf{N}=\{1,2, \ldots\}$ such that $\lambda(v)^{p} \leqslant$ $(\lambda \wedge K \mu)(v)^{p}+\mu(v)^{p-1} N^{-1}$ for each $v \in D$. Therefore

$$
\begin{aligned}
\sum_{D} \lambda(v)^{p} \mu(v)^{1-p} & \leqslant \sum_{D}\left[(\lambda \wedge K \mu)(v)^{p}+\mu(v)^{p-1} N^{-1}\right] \mu(v)^{1-p} \\
& \leqslant\|\lambda \wedge K \mu\|_{p}^{p}+1,
\end{aligned}
$$

and we have

LEMMA 2.1. If $\lambda \in A_{\mu}^{+}$and $1 \leqslant p \leqslant \infty$, then $\lambda \in H_{p}(\mu)$ iff $\left\{\|\lambda \wedge K \mu\|_{p} \mid K\right.$ $=1,2, \ldots\}$ is bounded.

We will denote by $H_{\omega}(\mu)$ the intersection of $H_{p}(\mu)$ for $1 \leqslant p<\infty$. The $\omega$-topology will be the smallest topology on $H_{\omega}(\mu)$ which contains each relative $p$-norm topology.

LEMMA 2.2. If $\left(a_{p}\right)_{p=1}^{\infty}$ is a sequence of positive numbers such that $\sum_{p=1}^{\infty} a_{p}\|\xi\|_{p}$ exists for each $\xi$ in $H_{\omega}(\mu)$, then the norm defined by $\|\xi\|=$ $\sum_{p=1}^{\infty} a_{p}\|\xi\|_{p}$ is complete.

Proof. Let $\left(\xi_{n}\right)$ be a $\|\cdot\|$ Cauchy sequence in $H_{\omega}(\mu)$. Then by 2.L.II we have

$$
\left\|\left|\xi_{n}\right|-\left|\xi_{m}\right|\right\|=\left\||| \xi_{n}|-| \xi_{m}||\right\| \leqslant\left\|\left|\xi_{n}-\xi_{m}\right|\right\|=\left\|\xi_{n}-\xi_{m}\right\|
$$

so that $\left(\left|\xi_{n}\right|\right)$ is also $\|\cdot\|$ Cauchy. Let $\left(\delta_{i}\right)=\left(\left|\xi_{n_{i}}\right|\right)$ be a subsequence of $\left(\left|\xi_{n}\right|\right)$ 
such that if $i$ and $r$ are positive integers then $\left\|\delta_{i}-\delta_{i+r}\right\|<1 / 2^{i}$. Let $\eta_{1}=\delta_{1}$ and for each $1<i \in \mathbf{N}$ let $\eta_{i}=\delta_{i} \bigvee \eta_{i-1}$. Then

$$
0 \leqslant \eta_{i+1}-\eta_{i}=\left(\delta_{i+1}-\eta_{i}\right)^{+} \leqslant\left(\delta_{i+1}-\delta_{i}\right)^{+} \leqslant\left|\delta_{i+1}-\delta_{i}\right|,
$$

so. that again by 2.L.II, $\left\|\eta_{i+1}-\eta_{i}\right\| \leqslant\left\|\delta_{i+1}-\delta_{i}\right\|<1 / 2^{i}$. Now for each $i$, $r \in \mathbf{N}$ we have

$$
\left\|\eta_{i+r}-\eta_{i}\right\| \leqslant \sum_{j=1}^{r}\left\|\eta_{i+j}-\eta_{i+j-1}\right\|<\sum_{j=1}^{r} \frac{1}{2^{i+j-1}}<\frac{1}{2^{i-1}}
$$

and, therefore, $\left(\eta_{i}\right)$ is $\|\cdot\|$ Cauchy and $\eta_{i} \leqslant \eta_{i+1}$ for each $i \in \mathbf{N}$.

Now any norm Cauchy sequence is $p$-norm Cauchy for each $1 \leqslant p<\infty$ and, hence, has a $p$-norm limit which (by the comparability of $p$-norms) is independent of $p$. Therefore there exists $\xi$ and $\eta$ in $H_{\omega}(\mu)$ such that $\eta_{i} \rightarrow^{p} \eta$ and $\xi_{n} \rightarrow^{p} \xi$ for each $1 \leqslant p<\infty$.

Now let $c>0$ and $K \in N$ be such that $\sum_{K+1}^{\infty} a_{p}\|\eta\|_{p}<c / 4$ and $\sum_{K+1}^{\infty} a_{p}\|\xi\|_{p}<c / 4$. For each $p \leqslant K$ let $N_{p}$ be such that if $i>N_{p}$, then $a_{p}\left\|\xi-\xi_{n_{i}}\right\|_{p}<c / 2 K$. Let $N=\max \left\{N_{1}, N_{2}, \ldots, N_{K}\right\}$ and $i>N$. Then

$$
\begin{aligned}
\left\|\xi-\xi_{n_{i}}\right\| & =\sum_{p=1}^{\infty} a_{p}\left\|\xi-\xi_{n_{i}}\right\|_{p} \\
& =\sum_{p=1}^{K} a_{p}\left\|\xi-\xi_{n_{i}}\right\|_{p}+\sum_{K+1}^{\infty} a_{p}\left\|\xi-\xi_{n_{i}}\right\|_{p} \\
& \leqslant \sum_{p=1}^{K} \frac{c}{2 K}+\sum_{K+1}^{\infty} a_{p}\left(\|\xi\|_{p}+\left\|\xi_{n_{i}}\right\|_{p}\right) \\
& \leqslant \frac{c}{2}+\frac{c}{4}+\sum_{K+1}^{\infty} a_{p}\left\|\delta_{i}\right\|_{p} \\
& \leqslant \frac{3 c}{4}+\sum_{K+1}^{\infty} a_{p}\left\|\eta_{i}\right\|_{p} \\
& \leqslant \frac{3 c}{4}+\sum_{K+1}^{\infty} a_{p}\|\eta\|_{p}<c .
\end{aligned}
$$

Therefore, $\xi_{n_{i}} \rightarrow^{\|\|} \| \xi$ and, hence, $\xi_{n} \rightarrow^{\|\|} \xi$, so that \|\| is complete.

3. A differential equivalence theorem for Hellinger integrals. In this section we will show that $H_{2^{k}}(\mu)$ is the " $K$ th image" of a certain function. The integral considered below is the refinement limit of sums over finite subdivisions of $S$ by elements of $F$. For further details see [1].

TheOREM 3.H. If $\xi \in \mathrm{ba}(F)$, then $\xi \in A_{\mu}^{+}$iff there exists an $\eta \in H_{2}(\mu)$ such that $\xi=\int \eta^{2} / \mu$.

The proof of this theorem of Hellinger [8] (for interval functions) carries over to our setting and suggests the function

$$
T: H_{2}^{+}(\mu) \rightarrow A_{\mu}^{+}: \eta \rightarrow \int \eta^{2} / \mu .
$$


The $\eta$ of Theorem 3.H is given by the function $R(\xi)=\int(\xi, \mu)^{1 / 2}$ which has the following properties [3]. $R$ is defined on all of ba(F) ${ }^{+}$and its restriction to $A_{\mu}^{+}$is the inverse of $T$. If $\xi \in \mathrm{ba}(F)^{+}$, then $R(\xi) \in H_{2}^{+}(\mu)$ and $T(R(\xi))=$ $\mathbf{a}_{\mu}(\xi)$ (the absolutely continuous part of $\xi$ ).

Lemma 3.1. Suppose $\alpha$ : $F \rightarrow \mathbf{R}^{+}, \mu(I)=0$ implies $\alpha(I)=0$ for each $I \in F$, $\Sigma_{D} \alpha(I)$ is nondecreasing for successive refinements and $\int_{S} \alpha(I)$ exists. Then $\int \alpha^{2} / \mu$ exists iff $\int\left(\int \alpha\right)^{2} / \mu$ exists, in which case they are equal.

Proof. If $\int\left(\int \alpha\right)^{2} / \mu$ exists, then, since $\Sigma_{D} \alpha^{2}(I) / \mu(I)$ is nondecreasing for successive refinements and bounded by $\int_{S}\left(\int_{v} \alpha(I)\right)^{2} / \mu(v)$, we have $\int \alpha^{2} / \mu$ exists.

If $\int \alpha^{2} / \mu$ exists, then

$$
\begin{aligned}
\int \alpha & =\int\left(\mu \alpha^{2} / \mu\right)^{1 / 2}=\int\left(\mu \int \alpha^{2} / \mu\right)^{1 / 2} \quad(\text { see }[1]) \\
& =R\left(\int \alpha^{2} / \mu\right) \in H_{2}(\mu),
\end{aligned}
$$

i.e. $\int\left(\int \alpha\right)^{2} / \mu$ exists.

If both exist, then

$$
\int \alpha^{2} / \mu \leqslant \int\left(\int \alpha\right)^{2} / \mu=T\left(\int \alpha\right)=T\left(R\left(\int \alpha^{2} / \mu\right)\right)=a_{\mu}\left(\int \alpha^{2} / \mu\right) \leqslant \int \alpha^{2} / \mu .
$$

For $\xi \in \mathrm{ba}(F)$ and $p \geqslant 1$ we have $\Sigma_{D}|\xi(I)|^{p} \mu(I)^{1-p}$ is nondecreasing for successive refinements [9] so that, for $\xi \in A_{\mu}, \xi \in H_{p}(\mu)$ iff $\int|\xi|^{p} \mu^{1-p}$ exists. Therefore by 3.1 and induction we have, for $\xi \in A_{\mu}^{+}, T^{K}(\xi)$ exists iff $\xi \in H_{2^{\kappa}}(\mu)$. Hence $R^{K}\left(A_{\mu}^{+}\right)=H_{2^{k}}^{+}(\mu)$.

4. The normability of $H_{\omega}(\mu)$. In the countably additive case the equivalence of (2) and (3) below can be found in [5].

THEOREM 4.1. These are equivalent:

(1) The w-topology is contained in a norm topology.

(2) The w-topology is normable.

(3) There exists a $p(1 \leqslant p<\infty)$ such that $H_{p}(\mu)=H_{\omega}(\mu)$.

(4) $A_{\mu}=H_{2}(\mu)$.

(5) $A_{\mu}=H_{\infty}(\mu)$.

(6) $A_{\mu}$ is finite dimensional.

$(1) \rightarrow(2)$. Suppose $\||\cdot|\|$ is a norm on $H_{\omega}(\mu)$ and its topology contains the $\omega$-topology. Then for each $p \in \mathbf{N}$ there exists an $M_{p}>0$ such that $\|\xi\|_{p} \leqslant$ $M_{p}\|\xi \mid\|$ for each $\xi \in H_{\omega}(\mu)$ and, therefore, $\|\cdot\|=\sum_{p=1}^{\infty}\|\cdot\|_{p} 2^{-p} M_{p}^{-1}$ is a complete norm by Lemma 2.2. The $\omega$-topology, being defined by a countable collection of norms, is a complete linear metric topology which is contained in the $\|\cdot\|$ topology and, hence, equal to it by the open mapping theorem [7].

$(2) \rightarrow(3)$. Suppose the $\omega$-topology is normable, then there exists a neighborhood $U$ of 0 which is $\omega$-bounded in the following sense: if $\left(\eta_{n}\right) \subseteq U$ and 
$\left(\alpha_{n}\right)$ is a null sequence, then $\left(\alpha_{n} \eta_{n} \rightarrow^{\omega} 0\right)$. Since the $\omega$-topology is generated by the $p$-norms, $p \in N$, there exists a $p \geqslant 1$ such that if $B=\left\{\eta \in H_{p}(\mu) \mid\right.$ $\left.\|\eta\|_{p} \leqslant 1\right\}$, then $B \cap H_{\omega}(\mu)$ is $\omega$-bounded. Now let $\xi \in B^{+}, r>p$ and $\xi_{K}=\xi \wedge K \mu$ for each $K \in \mathbf{N}$. Then $\left\|\xi_{K}\right\|_{r}$ is bounded, since otherwise we would have $\left(1 /\left\|\xi_{K}\right\|_{r}\right)^{1 / 2} \rightarrow 0$ and, therefore, $\left(1 /\left\|\xi_{K}\right\|_{r}\right)^{1 / 2} \xi_{K} \rightarrow^{\omega} 0$, hence $\left(1 /\left\|\xi_{K}\right\|_{r}\right)^{1 / 2} \xi_{K} r$-norm convergent to 0 , which is a contradiction. Therefore, by Lemma $2.1, \xi \in H_{r}(\mu)$ and, hence, $H_{p}(\mu)=H_{\omega}(\mu)$.

(3) $\rightarrow$ (4). Suppose $p \geqslant 1$ and $H_{\omega}(\mu)=H_{p}(\mu)$. By 2.L. it is sufficient to consider $A_{\mu}^{+}$. Let $K \in \mathrm{N}$ be such that $p<2^{K}$, then by $\S 3$ we have

$$
\begin{aligned}
A_{\mu}^{+} & =T^{K}\left(R^{K}\left(A_{\mu}^{+}\right)\right)=T^{K}\left(H_{2^{K}}^{+}(\mu)\right)=T^{K}\left(H_{2^{k+1}}^{+}(\mu)\right) \\
& =T^{K}\left(R^{K+1}\left(A_{\mu}^{+}\right)\right)=R\left(A_{\mu}^{+}\right)=H_{2}^{+}(\mu) .
\end{aligned}
$$

We will need the following to prove that (4) implies (5).

THEOREM 4.B. If each of $\lambda$ and $\mu$ is in $\mathrm{ba}(F)^{+}$, then $\lambda \in H_{\infty}(\mu)$ iff $H_{2}^{+}(\lambda) \subseteq H_{2}(\mu)$.

Proof. [4].

(4) $\rightarrow$ (5). Suppose $H_{2}(\mu)=A_{\mu}$ and $\lambda \in A_{\mu}^{+}$. Then $H_{2}(\lambda) \subseteq A_{\lambda} \subseteq A_{\mu}=$ $H_{2}(\mu)$ and, therefore, by $4 . B, \lambda \in H_{\infty}(\mu)$.

(5) $\rightarrow(6)$. Suppose $A_{\mu}$ is not finite dimensional; then there exists a disjoint sequence, $\left(v_{n}\right)$, on which $\mu$ is positive. Let $\left(a_{n}\right)$ be an unbounded sequence of positive numbers such that $\sum_{n=1}^{\infty} a_{n} \mu\left(v_{n}\right)<\infty$. Then $\sum_{n=1}^{\infty} a_{n} \mu_{n}$ is in $A_{\mu}$ but not $H_{\infty}(\mu)$, where $\mu_{n}$ is the contraction of $\mu$ to $v_{n}$, i.e. $\mu_{n}(I)=\mu\left(I \cap v_{n}\right)$ for each $I \in F$.

$(6) \rightarrow(1)$. This follows from the fact that $\|\cdot\|_{\infty}$ is stronger than each relative $p$-norm.

We conclude by noting that as a consequence of the equivalence of (1), (2) and (5) we have the following "internal" characterization of the normability of $H_{\omega}(\mu)$.

COROllary 4.1. The w-topology is normable iff $H_{\omega}(\mu)=H_{\infty}(\mu)$.

ADDED IN PROOF. For countably additive $\mu$ on a $\sigma$-field the property $H_{\omega}(\mu)=H_{\infty}(\mu), 4.1 .5,4.1 .6$ and several other conditions have been considered by Professor Ion Chitescu in Finitely purely atomic measures and $L^{P}$-spaces, Anal. Univ. Bucureşti Sti. Natur. 24 (1975), 23-29, MR 52 \#8366.

\section{BIBLIOGRAPHY}

1. W. D. L. Appling, Summability of real-valued set functions, Riv. Math. Univ. Parma 8 (1967), 77-100.

2. Richard Arens, The space $L^{\omega}$ and convex topological rings, Bull. Amer. Math. Soc. 7 (1946), 931-935.

3. Wayne C. Bell, A characterization theorem for the nearest point mappings of linear $C$-sets, Ann. Mat. Pura Appl. (to appear).

4. , A Hellinger integral characterization of Lipschitz functions, Boll. Un. Mat. Ital. (to appear). 
5. Henry W. Davis, F. J. Murray and J. K. Weber, Families of $L_{p}$-spaces with inductive and projective topologies, Pacific J. Math. 34 (1970), 619-638.

6. Inductive and projective limits of $L_{p}$-spaces, Portugal. Math. 31 (1972), 21-29.

7. M. M. Day, Normed linear spaces, Springer-Verlag, New York and Berlin, 1973.

8. E. Hellinger, Die Orthogonalinvarionten Quadratischer Formen von unendlichnielen Variablen, Dissertation, Gottingen, 1907.

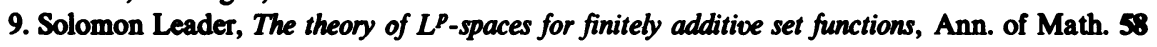
(1953), 528-543.

Department of Mathematics, Murray State University, MURray, Kentucky 42071 